\title{
Finite time singularities in a class of hydrodynamic models
}

\author{
V. P. Ruban ${ }^{1,2 *}$, D. I. Podolsky ${ }^{1}$, and J. J. Rasmussen ${ }^{2}$ \\ ${ }^{1}$ L.D. Landau Institute for Theoretical Physics, 2 Kosygin Street, 117334 Moscow, Russia \\ ${ }^{2}$ Optics and Fluid Dynamics Department, OFD-128, Risø National Laboratory, DK-4000 Roskilde, Denmark
}

November 5, 2018

\begin{abstract}
Models of inviscid incompressible fluid are considered, with the kinetic energy (i.e., the Lagrangian functional) taking the form $\mathcal{L} \sim \int k^{\alpha}\left|\mathbf{v}_{\mathbf{k}}\right|^{2} d^{3} \mathbf{k}$ in 3D Fourier representation, where $\alpha$ is a constant, $0<\alpha<1$. Unlike the case $\alpha=0$ (the usual Eulerian hydrodynamics), a finite value of $\alpha$ results in a finite energy for a singular, frozenin vortex filament. This property allows us to study the dynamics of such filaments without the necessity of a regularization procedure for short length scales. The linear analysis of small symmetrical deviations from a stationary solution is performed for a pair of anti-parallel vortex filaments and an analog of the Crow instability is found at small wave-numbers. A local approximate Hamiltonian is obtained for the nonlinear long-scale dynamics of this system. Self-similar solutions of the corresponding equations are found analytically. They describe the formation of a finite time singularity, with all length scales decreasing like $\left(t^{*}-t\right)^{1 /(2-\alpha)}$, where $t^{*}$ is the singularity time.
\end{abstract}

PACS numbers: 47.15.Ki, 47.32.Cc

\section{Introduction}

The question of the possibility for the spontaneous formation of a finite time singularity in solutions of the Euler equation for an ideal incompressible fluid has been discussed for a long time. At present, this fundamental problem of fluid dynamics is still far from a complete solution, though some rigorous analytical results have been obtained. So, it has been found by Beale, Kato and Majda [1], that no singularity occurs if the time-integral of the maximum of the vorticity magnitude is finite. Another result (Constantin and co-workers, [2]-[3]) postulates that a blow-up of the vorticity, if does take place, must be accompanied by a singularity in the field of the vorticity direction. In general, the nature of the presumed singularity has not yet been clarified, although many theoretical scenarios for blow-up have

*Electronic address: ruban@itp.ac.ru 
been suggested until now, and also extensive numerical simulations have been performed to observe the singular behavior (see [4]-[13] and references therein). In particular, the locally self-similar regime of singularity formation seems very probable. For this regime, a region of finite size may be distinguished in flow, where all length scales, corresponding to vorticity distribution, decrease like $\left(t^{*}-t\right)^{1 / 2}$, the velocity increases according to $\left(t^{*}-t\right)^{-1 / 2}$, and the maximum of the vorticity behaves like $\left(t^{*}-t\right)^{-1}$. It is necessary to emphasize that this is the only possible scaling, which is compatible both with the dimensional structure of the Euler equation and with the freezing-in property of the vorticity. In this process, an accelerated straining of vortex lines takes place, and it is the reason for amplification of the vorticity magnitude. It is very important that the curvature of vortex lines in the assumed self-similar solutions should tend to infinity in the vicinity of the singular point, in accordance with the result of Constantin, Fefferman and Majda. Thus, the problem of singularity in the curvature of vortex lines and the problem of singularity in their local stretching are closely connected.

In this paper, we take the point of view that infinite curvature of frozen-in vortex lines is in some sense a more fundamental characteristics of hydrodynamic singularity than infinite value of the vorticity maximum. To illustrate this statement, we consider a class of models of an incompressible inviscid fluid, different from Eulerian hydrodynamics, such that finite energy solutions with infinitely thin frozen-in vortex filaments of finite strengths are possible. Thus, we deal with a situation when the vorticity maximum is infinite from the very beginning, but nevertheless, this fact itself does not imply a singular behaviour in the dynamics of vortex strings, while their shape is smooth and the distance between them is finite. However, the interaction between filaments may result in formation of a finite time singularity for the curvature of vortex strings. It is the main purpose of present work to study this phenomenon analytically.

It is a well known fact that absence of solutions with singular vortex filaments in Eulerian hydrodynamics is manifested, in particular, as a logarithmic divergency of the corresponding formal expression for the energy functional of an infinitely thin vortex filament having a finite circulation $\Gamma$ and a shape $\mathbf{R}(\xi)$ (this is actually the Hamiltonian functional determining entirely the dynamics of the system, as is shown in Section 2):

$$
\mathcal{H}^{\Gamma}\{\mathbf{R}(\xi)\}=\frac{\Gamma^{2}}{8 \pi} \oint \oint \frac{\left(\mathbf{R}^{\prime}\left(\xi_{1}\right) \cdot \mathbf{R}^{\prime}\left(\xi_{2}\right)\right) d \xi_{1} d \xi_{2}}{\left|\mathbf{R}\left(\xi_{1}\right)-\mathbf{R}\left(\xi_{2}\right)\right|} \rightarrow \infty .
$$

More important is that the self-induced velocity of a curved string in Eulerian hydrodynamics is also infinite. This is the reason, why we cannot work in the framework of Eulerian hydrodynamics with such one-dimensional objects, that are very attractive for theoretical treatment. The situation becomes more favourable, when we consider a class of regularized models, with the divergency of the energy functional eliminated. It should be stressed here that in regularized systems the usual relation $\Omega=$ curl $\mathbf{v}$ between the vorticity and velocity fields is no more valid, and in this case $\Gamma$ is not the circulation of the velocity around the filament, but it is the circulation of the canonical momentum field (see Section 2 for more details). However, dynamical properties of a de-singularized system depend on the manner of regularization. For instance, it is possible to replace the singular Green's function $G\left(\left|\mathbf{R}_{1}-\mathbf{R}_{2}\right|\right.$ ) in (1) (where $G(r) \sim 1 / r$ ) by some analytical function which has no singular points near the real axis in the complex plane (for examples by $G_{q}(r) \sim \tanh (q r) / r$ or by 
$\left.G_{\epsilon}(r) \sim 1 / \sqrt{r^{2}+\epsilon^{2}}\right)$. In that case we may not expect any finite time singularity formation, because the corresponding velocity field created by the vortex string appears to be too smooth with any shape of the curve, and this fact prevents drawing together some pieces of the string. With such a very smooth velocity field, a singularity formation needs an infinite time.

In this paper we consider another type of regularization of the Hamiltonian functional, when the Green's function is still singular, but this singularity is integrable in the contour integral analogous to the expression (11):

$$
\mathcal{H}_{\alpha}^{\Gamma}\{\mathbf{R}(\xi)\} \sim \frac{\Gamma^{2}}{2} \oint \oint \frac{\left(\mathbf{R}^{\prime}\left(\xi_{1}\right) \cdot \mathbf{R}^{\prime}\left(\xi_{2}\right)\right) d \xi_{1} d \xi_{2}}{\left|\mathbf{R}\left(\xi_{1}\right)-\mathbf{R}\left(\xi_{2}\right)\right|^{1-\alpha}},
$$

with a small but finite positive constant $0<\alpha \ll 1$. If $\alpha$ is not small, we actually have models that are rather different from Eulerian hydrodynamics. Nevertheless, such models still have many common features with usual hydrodynamics, which are important for singularity formation in the process of the interaction between vortex filaments: a similar hydrodynamic type structure of the Hamiltonian and a power-like behaviour of the Green's function, with negative exponent. Therefore we believe that it is useful to investigate these models, especially the question about the formation of a finite time singularity in the vortex line curvature. We hope the results of our study will shed more light on the problem of blow-up in Eulerian hydrodynamics.

This paper is organized as follows. In Section II, we briefly review some basic properties of frozen-in vorticity dynamics in a perfect fluid, with giving necessary definitions for theoretical conceptions used in our study. In general, our approach is based on the Hamiltonian formalism for frozen-in vortex lines [14]-17]. Then, in Section III, we perform the linear analysis of stability for a pair of symmetric anti-parallel vortex filaments and find an instability at small wave numbers analogous to the Crow instability [18. In Section IV, we postulate a local approximate Hamiltonian for the long scale nonlinear dynamics of the pair of filaments and present analytical self-similar solutions of the corresponding equations. Those solutions describe finite time singularity formation, with the length scales decreasing like $\left(t^{*}-t\right)^{1 /(2-\alpha)}$, and this is the main result of present work. In Section $\mathrm{V}$, we make some concluding remarks about vortex filaments of a finite width, about long scale approximation for systems with the Green's function of a general form, and finally about how it is possible to improve the approximation in the case of small $\alpha$, when the unstable region is narrow in wave number space. In Appendix A, we write in terms of the special mathematical functions some integral expressions needed for calculation of instability increment of the vortex pair. In Appendix B, we provide details about the integration procedure for the system of ordinary differential equations related to the self-similar solutions.

\section{Hamiltonian dynamics of vortex filaments}

To clarify the meaning of the suggested models (21) and to explain the employed theoretical method, we recall some general properties of frozen-in vorticity dynamics in a perfect fluid, starting from the Lagrangian formalism [19]- 25], [14]-17]. 
Let a Lagrangian functional $\mathcal{L}\{\mathbf{v}\}$ specify the dynamics of some incompressible medium of unit density, with the solenoidal velocity field $\mathbf{v}(\mathbf{r}, t)$. Especially we are interested here in systems with quadratic Lagrangians, which in 3D Fourier representation take the form:

$$
\mathcal{L}_{M}\{\mathbf{v}\}=\frac{1}{2} \int \frac{d^{3} \mathbf{k}}{(2 \pi)^{3}} M(k)\left|\mathbf{v}_{\mathbf{k}}\right|^{2},
$$

where $M(k)$ is some given positive function of the absolute value of the wave vector $\mathbf{k}$. This expression should be understood as the kinetic energy on the group of volume preserving mappings $\mathbf{x}(\mathbf{a}, t)$, and the velocity field $\mathbf{v}(\mathbf{x}, t)$ is defined as the time derivative $\dot{\mathbf{x}}(\mathbf{a}, t)$ taken at the point $\mathbf{a}(\mathbf{x}, t)$. Obviously, all the systems (3) possess the properties of homogeneity and isothropy in the space. It is clear that the usual Eulerian hydrodynamics corresponds to the simplest case $M(k)=1$. Another physically important example concerns the homogeneous incompressible electron magnetohydrodynamics (EMHD), for which $M(k)=1+q^{2} / k^{2}$, with a constant $q$ beeng the screening parameter [17], [26]. Also the case $M(k)=1+\lambda^{2} k^{2}$ has been studied, with a constant $\lambda$, which corresponds to the so called avereged Eulerian hydrodynamics (see, for instance, the papers [27], [28] for more details). In the general case, the systems (5) may be understood as models for some inviscid non-newtonian fluids. It should be noted that there exists a direct relation between such models and the vortex blob method introduced by Chorin for desingularization of the Eulerian hydrodynamics [29]. Some discussion of this relation, for the case of the averaged Eulerian hydrodynamics, can be found in the papers [30] and [31].

Due to the presence of the Noether type symmetry with respect to relabeling of Lagrangian labels of fluid points [21]-[25], [14]-[16], all such systems have an infinite number of integrals of motion, which can be expressed as conservation of the circulations $\Gamma_{c}$ of the canonical momentum field $\mathbf{p}(\mathbf{r}, t)$,

$$
\mathbf{p}=\frac{\delta \mathcal{L}}{\delta \mathbf{v}},
$$

along any closed contour $c(t)$ advected by flow, thus the generalized theorem of Kelvin is valid:

$$
\Gamma_{c}=\oint_{c(t)}(\mathbf{p} \cdot d \mathbf{l})=\text { const. }
$$

These integrals of motion correspond to the frozen-in property of the canonical vorticity field $\Omega(\mathbf{r}, t)$

$$
\boldsymbol{\Omega} \equiv \operatorname{curl} \mathbf{p}=\operatorname{curl} \frac{\delta \mathcal{L}}{\delta \mathbf{v}} .
$$

After defining the Hamiltonian functional $\mathcal{H}\{\Omega\}$,

$$
\mathcal{H}\{\boldsymbol{\Omega}\}=\left.\left(\int\left(\frac{\delta \mathcal{L}}{\delta \mathbf{v}} \cdot \mathbf{v}\right) d \mathbf{r}-\mathcal{L}\right)\right|_{\mathbf{v}=\mathbf{v}\{\boldsymbol{\Omega}\}},
$$

the equation of motion for the vorticity takes the form

$$
\Omega_{t}=\operatorname{curl}\left[\operatorname{curl}\left(\frac{\delta \mathcal{H}}{\delta \Omega}\right) \times \Omega\right] .
$$


This equation describes the transport of frozen-in vortex lines by the flow having the velocity field

$$
\mathbf{v}=\operatorname{curl}\left(\frac{\delta \mathcal{H}}{\delta \Omega}\right) .
$$

It is very important in this process that all topological characteristics of the vorticity field are conserved [19], 32], 33]. It follows from Eqs. (33), (6) and (77), that the Hamiltonian $\mathcal{H}_{M}$ corresponding to the Lagrangian $\mathcal{L}_{M}$ is

$$
\mathcal{H}_{M}\{\boldsymbol{\Omega}\}=\frac{1}{2} \int \frac{d^{3} \mathbf{k}}{(2 \pi)^{3}} \frac{\left|\mathbf{\Omega}_{\mathbf{k}}\right|^{2}}{k^{2} M(k)}=\frac{1}{2} \iint G_{M}\left(\left|\mathbf{r}_{1}-\mathbf{r}_{2}\right|\right)\left(\boldsymbol{\Omega}\left(\mathbf{r}_{1}\right) \cdot \boldsymbol{\Omega}\left(\mathbf{r}_{2}\right) d \mathbf{r}_{1} d \mathbf{r}_{2},\right.
$$

with the Green's function $G_{M}(r)$ being equal to the following integral:

$$
G_{M}(r)=\int \frac{d^{3} \mathbf{k}}{(2 \pi)^{3}} \frac{e^{i \mathbf{k r}}}{k^{2} M(k)}=\frac{1}{2 \pi^{2}} \int_{0}^{+\infty} \frac{\sin k r}{k r} \frac{d k}{M(k)} .
$$

The frozen-in vorticity field can be represented in topologically simple cases as a continuous distribution of vortex lines [14]-[17]:

$$
\boldsymbol{\Omega}(\mathbf{r}, t)=\int_{\mathcal{N}} d^{2} \nu \oint \delta(\mathbf{r}-\mathbf{R}(\nu, \xi, t)) \frac{\partial \mathbf{R}}{\partial \xi} d \xi,
$$

where a 2D Lagrangian coordinate $\nu=\left(\nu_{1}, \nu_{2}\right)$, which lies in some manifold $\mathcal{N}$, is the label of a vortex line, while the longitudinal coordinate $\xi$ determines a point on the line.

The important characteristics of the system: the (virtual) linear momentum $\mathbf{P}$ and the angular momentum $\mathbf{M}$ can be expressed as follows:

$$
\begin{aligned}
\mathbf{P} & =\int_{\mathcal{N}} d^{2} \nu \frac{1}{2} \oint\left[\mathbf{R} \times \mathbf{R}_{\xi}\right] d \xi, \\
\mathbf{M} & =\int_{\mathcal{N}} d^{2} \nu \frac{1}{3} \oint\left[\mathbf{R} \times\left[\mathbf{R} \times \mathbf{R}_{\xi}\right]\right] d \xi .
\end{aligned}
$$

In the limit, when the shapes $\mathbf{R}(\nu, \xi, t)$ of vortex lines do not depend on the label $\nu$, we have one singular vortex filament with a finite circulation $\Gamma=\int_{\mathcal{N}} d^{2} \nu$. In this case, the flow is potential in the space around the filament: $\mathbf{p}=\nabla \Phi$, with a multi-valued scalar potential $\Phi(\mathbf{r}, t)$. The potential flow domain is passive from the dynamical viewpoint, because there the flow depends entirely on the filament shape. The dynamics of the shape $\mathbf{R}(\xi, t)$ of such infinitely thin vortex filament is determined in a self-consistent manner by the variational principle with the Lagrangian $\mathcal{L}_{M}^{\Gamma}\{\mathbf{R}\}$ [14]- [17],

$$
\mathcal{L}_{M}^{\Gamma}=\Gamma \oint\left(\left[\mathbf{R}^{\prime} \times \mathbf{R}_{t}\right] \cdot \mathbf{D}(\mathbf{R})\right) d \xi-\frac{\Gamma^{2}}{2} \oint \oint G_{M}\left(\left|\mathbf{R}\left(\xi_{1}\right)-\mathbf{R}\left(\xi_{2}\right)\right|\right)\left(\mathbf{R}^{\prime}\left(\xi_{1}\right) \cdot \mathbf{R}^{\prime}\left(\xi_{2}\right)\right) d \xi_{1} d \xi_{2},
$$

where the vector function $\mathbf{D}(\mathbf{R})$ must have unit divergence [17]:

$$
\operatorname{div}_{\mathbf{R}} \mathbf{D}(\mathbf{R})=1 .
$$


The generalization of the expression (15) to a case of several filaments with the circulations $\Gamma^{(n)}$ and shapes $\mathbf{R}^{(n)}(\xi, t), n=1 . . N$, is straightforward: one should write a single sum over $n$ for the first term and a double sum for the Hamiltonian.

It is easy to see that the Hamiltonian (2) corresponds to the function $M(k)$ in the form

$$
M(k) \sim k^{\alpha} .
$$

The choice of the longitudinal parameter $\xi$ is not unique, but this does not affect the dynamics of the vortex string which is an invariant geometric object. Sometimes it is convenient to use parameterization of the vortex line shape by the Cartesian coordinate:

$$
\mathbf{R}(\xi, t)=(X(\xi, t), Y(\xi, t), \xi) .
$$

Then the choice $\mathbf{D}=(0, Y, 0)$ gives immediately that $X(\xi, t)$ and $Y(\xi, t)$ are canonically conjugated quantities.

Hereafter, we will consider vortex filaments with unit circulation for simplicity. So the symbol $\Gamma$, if appearing in some expressions below, will mean the special mathematical Gamma function. Also, without loss of generality, all quantities may be considered as dimensionless.

Now, for some fixed value of the parameter $\alpha$, let us consider the symmetrical dynamics of a pair of oppositely rotating vortex filaments, with the symmetry plane $y=$ const. Due to this symmetry, it is sufficient to consider only one of the filaments. It follows from the above discussion that the exact expression for the Hamiltonian of this system is the following:

$$
\begin{aligned}
& \mathcal{H}_{\alpha}=\frac{1}{2} \iint \frac{\left(1+X_{1}^{\prime} X_{2}^{\prime}+Y_{1}^{\prime} Y_{2}^{\prime}\right) d \xi_{1} d \xi_{2}}{\left(\left(\xi_{1}-\xi_{2}\right)^{2}+\left(X_{1}-X_{2}\right)^{2}+\left(Y_{1}-Y_{2}\right)^{2}\right)^{\frac{1-\alpha}{2}}} \\
& +\frac{1}{2} \iint \frac{\left(-1-X_{1}^{\prime} X_{2}^{\prime}+Y_{1}^{\prime} Y_{2}^{\prime}\right) d \xi_{1} d \xi_{2}}{\left(\left(\xi_{1}-\xi_{2}\right)^{2}+\left(X_{1}-X_{2}\right)^{2}+\left(Y_{1}+Y_{2}+b\right)^{2}\right)^{\frac{1-\alpha}{2}}},
\end{aligned}
$$

where $b$ is the mean distance between the two filaments $(b$ does not depend on time because of the conservation law for the momentum (130), $X_{1}=X\left(\xi_{1}\right), X_{1}^{\prime}=X^{\prime}\left(\xi_{1}\right)$ and so on. The first term in Eq.(19) describes the non-local self-interaction of the filament, while the second one corresponds to the interaction with the second filament. The Hamiltonian equations of motion have the form

$$
\dot{X}(\xi)=\frac{\delta \mathcal{H}_{\alpha}}{\delta Y(\xi)}, \quad \dot{Y}(\xi)=-\frac{\delta \mathcal{H}_{\alpha}}{\delta X(\xi)} .
$$

\section{Crow instability for a pair of vortex filaments}

The system with the Hamiltonian (19) possesses the exact stationary solution

$$
X(\xi, t)=C(\alpha, b) t, \quad Y(\xi, t)=0,
$$

which describes the uniform motion of straight filaments. Here the stationary velocity $C(\alpha, b)$ is proportional to $b^{\alpha-1}$. But this solution appears to be unstable due to an analog of the 
Crow instability 18 . In this section we consider the linear evolution of small perturbations of the stationary solution, and derive the linear growth rate.

To perform the linear analysis of small deviations of the vortex shape from a straight line, we need the quadratic part of the Hamiltonian (19):

$$
\begin{gathered}
\mathcal{H}_{\alpha}^{(2)}=\frac{1}{2} \iint \frac{\left(X_{1}^{\prime} X_{2}^{\prime}+Y_{1}^{\prime} Y_{2}^{\prime}\right)}{\left|\xi_{1}-\xi_{2}\right|^{1-\alpha}} d \xi_{1} d \xi_{2}+\frac{1}{2} \iint\left(\frac{\alpha-1}{2}\right) \frac{\left[\left(X_{1}-X_{2}\right)^{2}+\left(Y_{1}-Y_{2}\right)^{2}\right]}{\left|\xi_{1}-\xi_{2}\right|^{3-\alpha}} d \xi_{1} d \xi_{2} \\
+\frac{1}{2} \iint \frac{\left(Y_{1}^{\prime} Y_{2}^{\prime}-X_{1}^{\prime} X_{2}^{\prime}\right)}{\left(\left(\xi_{1}-\xi_{2}\right)^{2}+b^{2}\right)^{\frac{1-\alpha}{2}}} d \xi_{1} d \xi_{2}-\frac{1}{2} \iint\left(\frac{\alpha-1}{2}\right) \frac{\left[\left(X_{1}-X_{2}\right)^{2}+\left(Y_{1}+Y_{2}\right)^{2}\right]}{\left(\left(\xi_{1}-\xi_{2}\right)^{2}+b^{2}\right)^{\frac{3-\alpha}{2}}} d \xi_{1} d \xi_{2} \\
-\frac{1}{2} \iint\left(\frac{\alpha-1}{2}\right)\left(\frac{\alpha-3}{2}\right) \frac{2 b^{2}\left(Y_{1}+Y_{2}\right)^{2}}{\left(\left(\xi_{1}-\xi_{2}\right)^{2}+b^{2}\right)^{\frac{5-\alpha}{2}}} d \xi_{1} d \xi_{2}
\end{gathered}
$$

For further consideration, it is useful to rewrite it in the 1D Fourier representation:

$$
\mathcal{H}_{\alpha}^{(2)}=\frac{1}{2} \int \frac{d k}{2 \pi}\left(A_{\alpha}(k) X_{k} X_{-k}+B_{\alpha}(k) Y_{k} Y_{-k}\right)
$$

Expressions for the functions $A_{\alpha}(k)$ and $B_{\alpha}(k)$ follow from Eq.(22). So, $A_{\alpha}(k)$ can be represented as follows:

$$
\begin{gathered}
A_{\alpha}(k)=2 k^{2} b^{\alpha} \int_{0}^{+\infty} \cos (k b \zeta)\left(\frac{1}{\zeta^{1-\alpha}}-\frac{1}{\left(\zeta^{2}+1\right)^{\frac{1-\alpha}{2}}}\right) d \zeta \\
+2(\alpha-1) b^{\alpha-2} \int_{0}^{+\infty}(1-\cos (k b \zeta))\left(\frac{1}{\zeta^{3-\alpha}}-\frac{1}{\left(\zeta^{2}+1\right)^{\frac{3-\alpha}{2}}}\right) d \zeta \\
=2(1-\alpha)^{2} b^{\alpha-2} \int_{0}^{+\infty}(1-\cos (k b \zeta))\left(\frac{1}{\zeta^{3-\alpha}}-\frac{1}{\left(\zeta^{2}+1\right)^{\frac{3-\alpha}{2}}}+\left(\frac{3-\alpha}{1-\alpha}\right) \frac{1}{\left(\zeta^{2}+1\right)^{\frac{5-\alpha}{2}}}\right) d \zeta
\end{gathered}
$$

Obviously, $A_{\alpha}(k)$ is positive everywhere. Analogous calculations for the function $B_{\alpha}(k)$ give:

$$
\begin{gathered}
B_{\alpha}(k)=2 k^{2} b^{\alpha} \int_{0}^{+\infty} \cos (k b \zeta)\left(\frac{1}{\zeta^{1-\alpha}}+\frac{1}{\left(\zeta^{2}+1\right)^{\frac{1-\alpha}{2}}}\right) d \zeta+2(\alpha-1) b^{\alpha-2} \int_{0}^{+\infty}(1-\cos (k b \zeta)) \frac{d \zeta}{\zeta^{3-\alpha}} \\
+2(1-\alpha) b^{\alpha-2} \int_{0}^{+\infty}(1+\cos (k b \zeta))\left(\frac{1}{\left(\zeta^{2}+1\right)^{\frac{3-\alpha}{2}}}+\frac{\alpha-3}{\left(\zeta^{2}+1\right)^{\frac{5-\alpha}{2}}}\right) d \zeta \\
=2(1-\alpha)^{2} b^{\alpha-2} \int_{0}^{+\infty}(1-\cos (k b \zeta)) \frac{d \zeta}{\zeta^{3-\alpha}} \\
-2(1-\alpha)(3-\alpha) b^{\alpha-2} \int_{0}^{+\infty}(1+\cos (k b \zeta))\left(\frac{2}{\left(\zeta^{2}+1\right)^{\frac{5-\alpha}{2}}}-\frac{1}{\left(\zeta^{2}+1\right)^{\frac{3-\alpha}{2}}}\right) d \zeta
\end{gathered}
$$

In Appendix $\mathrm{A}, A_{\alpha}(k)$ and $B_{\alpha}(k)$ are expressed through the Euler Gamma function $\Gamma(x)$ and the modified Bessel functions of the second kind $K_{\nu}(x)$. 


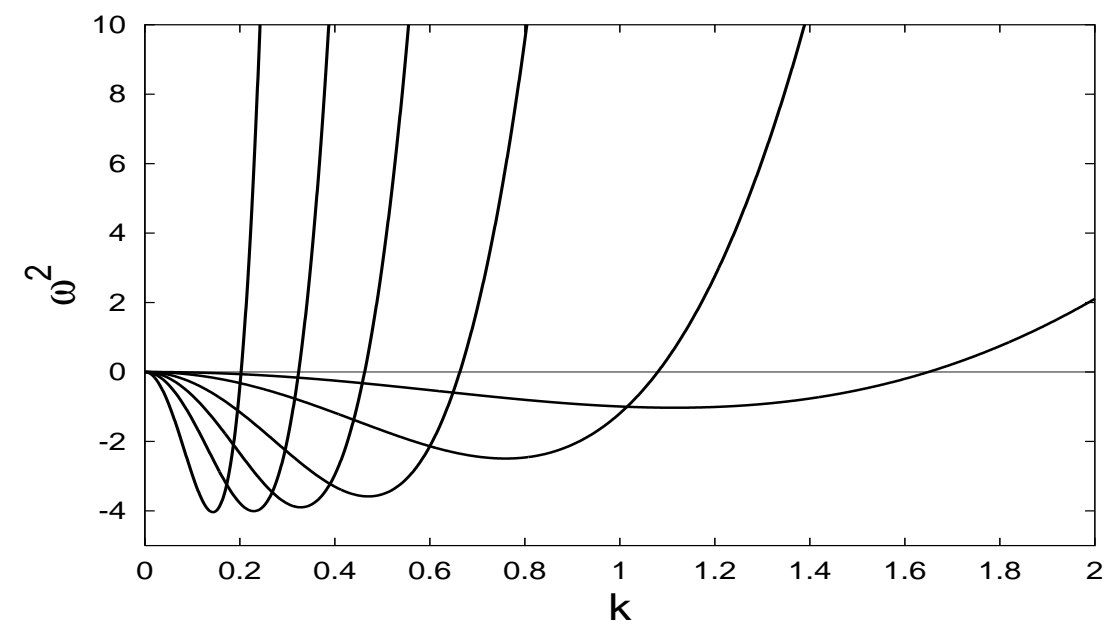

Figure 1: The dependences $\omega_{\alpha}^{2}(k)=A_{\alpha}(k) B_{\alpha}(k)$ with $b=1$ for $\alpha=0.01,0.025,0.05,0.1,0.25$, 0.5. Lines corresponding to the given values of $\alpha$ intersect the horizontal axis in the indicated order.

The dispersion relation between the frequency $\omega_{\alpha}$ of a small amplitude perturbation of the filament shape and the corresponding wave number $k$ is simply given by the formula

$$
\omega_{\alpha}^{2}(k)=A_{\alpha}(k) B_{\alpha}(k),
$$

since the linearized equations of motion for $X_{k}$ and $Y_{k}$ are

$$
\dot{X}_{k}=B_{\alpha}(k) Y_{k}, \quad \dot{Y}_{k}=-A_{\alpha}(k) X_{k},
$$

as follows from Eq.(20). In Fig.1] we have plotted $\omega_{\alpha}^{2}$ versus $k$ for several values of $\alpha$.

It is easy to see that at small wave numbers the product $A_{\alpha}(k) B_{\alpha}(k)$ is negative. Indeed, after some calculations we obtain in leading order for $k b \ll 1$ :

$$
\begin{gathered}
A_{\alpha}(k) \approx k^{2} b^{\alpha}\left(\frac{1-\alpha}{\alpha}\right) I_{3-\alpha}, \\
B_{\alpha}(k) \approx-4(1-\alpha)^{2} b^{\alpha-2} I_{3-\alpha},
\end{gathered}
$$

where the constant $I_{3-\alpha}$ is given by the integral

$$
I_{3-\alpha}=\int_{0}^{+\infty} \frac{d \zeta}{\left(\zeta^{2}+1\right)^{\frac{3-\alpha}{2}}}=\frac{\sqrt{\pi} \Gamma\left(1-\frac{\alpha}{2}\right)}{2 \Gamma\left(\frac{3-\alpha}{2}\right)},
$$

with $\Gamma$ (..) being the Gamma function. Therefore, an instability takes place at small $k$. The unstable domain in the wave number space corresponds to a range $|k| b<q_{0}(\alpha)$ where $B_{\alpha}(k)$ is negative, with the function $q_{0}(\alpha)$ behaving, at small values of $\alpha$, like $\sqrt{\alpha}$ :

$$
q_{0}(\alpha) \approx 2 \sqrt{\alpha}, \quad \alpha \ll 1 .
$$

The plot of $q_{0}(\alpha)$ is shown in Fig.2. The instability increment $\gamma_{\alpha}(k)=\sqrt{-A_{\alpha}(k) B_{\alpha}(k)}$ is 


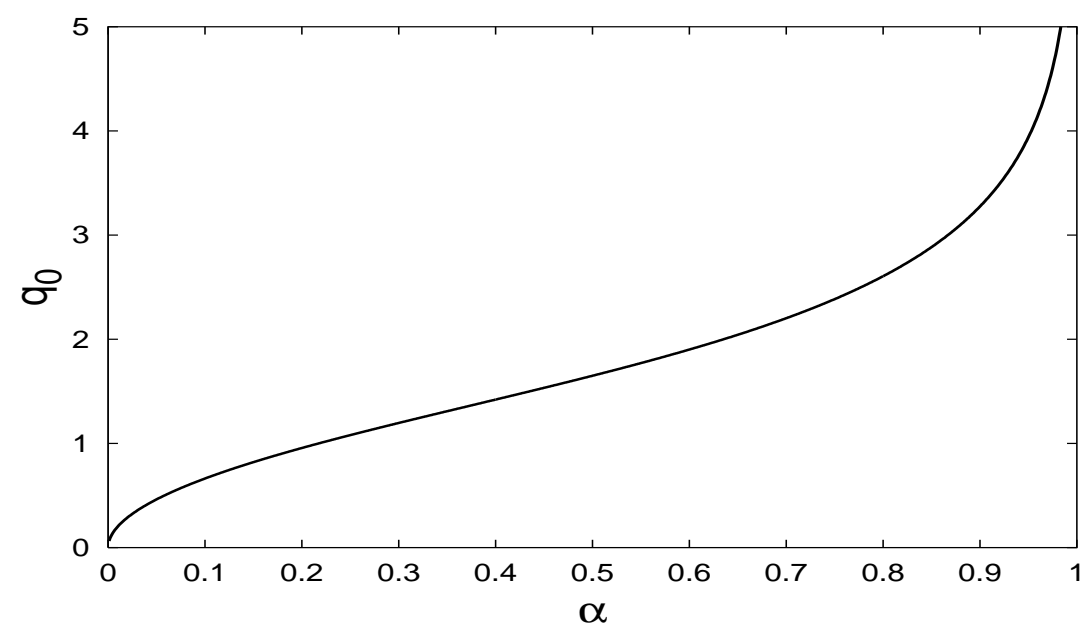

Figure 2: The boundary of instability $q_{0}(\alpha)$.

proportional to the absolute value of $k$ at very small values of $k b$ :

$$
\gamma(k) \approx(1-\alpha) I_{3-\alpha} \cdot 2|k| b^{\alpha-1} \sqrt{(1-\alpha) / \alpha} .
$$

However, for each $\alpha$ there exists a maximum value $\gamma_{\max }(\alpha)$ of the increment, which is attained at $k b \sim \sqrt{\alpha}$. Therefore the approximate expressions (29) and (32) are valid only if $|k| b \ll \sqrt{\alpha}$.

For large wave numbers, $|k| b \gg 1$, the functions $A_{\alpha}(k)$ and $B_{\alpha}(k)$ are both positive. The asymptotic approximations in that region are:

$$
A_{\alpha}(k) \approx B_{\alpha}(k) \approx 2(1-\alpha)^{2} k^{2-\alpha} \int_{0}^{+\infty} \frac{(1-\cos \eta)}{\eta^{3-\alpha}} d \eta=k^{2-\alpha} \frac{2(1-\alpha) \cos (\pi \alpha / 2) \Gamma(\alpha)}{2-\alpha} .
$$

Note that this expression does not contain the parameter $b$. For a single vortex filament it is actually the exact expression for $A_{\alpha}(k)$ and $B_{\alpha}(k)$, which is valid in the whole range of $k$.

A general nonlinear analysis of the non-local system (19) is difficult. Therefore we need some simplified model which would approximate the nonlinear dynamics, at least in the most interesting long scale unstable regime. In the next section, we suggest such an approximate model and find a class of solutions describing the formation of a finite time singularity.

\section{Singularity in long-scale nonlinear dynamics}

We note that the same long-scale limit as $(28-29)$ can be obtained from the local nonlinear Hamiltonian

$$
\mathcal{H}_{l}\{\mathbf{R}(\xi)\}=(1-\alpha) I_{3-\alpha} \oint \frac{(2 Y)^{\alpha}}{\alpha} \sqrt{X^{\prime 2}+Z^{\prime 2}} d \xi,
$$

where the coordinate $Y(\xi)$ is measured from the symmetry plane. This Hamiltonian approximates the exact non-local Hamiltonian of a symmetrical pair of vortex filaments in the case when the ratio of a typical value of $Y$ to a typical longitudinal scale $L$ is much smaller than $q_{0}(\alpha)$ :

$$
Y / L \ll \sqrt{\alpha}
$$


In particular, this means that the slope of the curve with respect to the symmetry plane should be small, and also $Y$ should be small in comparison with the radius of the line curvature. When $Y=$ const, $X^{\prime}=$ const, $Z^{\prime}=$ const, expression (34) gives the same result for uniform stationary motion as the exact Hamiltonian.

With the Cartesian parametrization (18), the corresponding approximate local nonlinear equations of motion have the form (after appropriate time rescaling)

$$
\begin{aligned}
& \dot{X}=\frac{1}{(2-\alpha)} \frac{\sqrt{1+X^{\prime 2}}}{Y^{1-\alpha}}, \\
& \dot{Y}=\frac{1}{(2-\alpha) \alpha}\left(\frac{Y^{\alpha} X^{\prime}}{\sqrt{1+X^{\prime 2}}}\right)^{\prime}
\end{aligned}
$$

and they allow to obtain a simple explanation of the instability. On a qualitative level of understanding, the reason for the instability is that if initially some pieces of the curve were more close to the symmetry plane and convex in the direction of motion, then at subsequent moments in time the curvature will be increasing because of smaller values of $Y$ and corresponding larger velocity, while $Y$ will be decreasing due to the curvature. Thus, the feedback is positive and the system is unstable. In the final stage of the instability development, a locally self-similar regime in the dynamics is possible, because the above equations admit the self-similar substitution

$$
\begin{aligned}
& X(\xi, t)=X^{*}-\left(t^{*}-t\right)^{\beta} x\left(\left(\xi-\xi^{*}\right)\left(t^{*}-t\right)^{-\beta}\right), \\
& Y(\xi, t)=\left(t^{*}-t\right)^{\beta} y\left(\left(\xi-\xi^{*}\right)\left(t^{*}-t\right)^{-\beta}\right),
\end{aligned}
$$

with arbitrary constants $X^{*}, \xi^{*}, t^{*}$, and with the exponent

$$
\beta=\frac{1}{2-\alpha}
$$

After substituting Eqs.(38-39) into Eqs.(36-37), we obtain a pair of ordinary differential equations for the functions $x(z)$ and $y(z)$ :

$$
\begin{aligned}
& x-z \cdot \frac{d x}{d z}=\frac{\sqrt{1+(d x / d z)^{2}}}{y^{1-\alpha}} \\
& y-z \cdot \frac{d y}{d z}=\frac{1}{\alpha} \cdot \frac{d}{d z}\left(\frac{y^{\alpha} \cdot(d x / d z)}{\sqrt{1+(d x / d z)^{2}}}\right)
\end{aligned}
$$

where $z=\left(\xi-\xi^{*}\right)\left(t^{*}-t\right)^{-\beta}$.

However, with this choice of parametrization of the curve, the obviously existing symmetry of the system (34) with respect to rotation in the $x-z$ plane is hidden. For taking advantage of this symmetry, cylindrical coordinates are more appropriate, with the angle coordinate $\varphi$ serving as the longitudinal parameter:

$$
(X, Y, Z)=(R(\varphi, t) \cos \varphi, Y(\varphi, t),-R(\varphi, t) \sin \varphi) .
$$


Instead of the equations of motion (36-37), we obtain the equivalent system (where a same time rescaling as in (36-37) is performed)

$$
\begin{aligned}
-(2-\alpha) R \dot{R} & =\frac{\sqrt{R^{2}+R^{\prime 2}}}{Y^{1-\alpha}} \\
-(2-\alpha) R \dot{Y} & =\frac{1}{\alpha}\left(\frac{Y^{\alpha} R^{\prime}}{\sqrt{R^{2}+R^{\prime 2}}}\right)^{\prime}-\frac{1}{\alpha} \frac{R Y^{\alpha}}{\sqrt{R^{2}+R^{\prime 2}}} .
\end{aligned}
$$

Here $(. .)^{\prime}=\partial_{\varphi}(.$.$) . This system follows from the Lagrangian written in cylindrical coordinates$

$$
\mathcal{L}_{\varphi} \sim \int\left((2-\alpha) \frac{R^{2}}{2} \dot{Y}-\frac{Y^{\alpha}}{\alpha} \sqrt{R^{2}+R^{\prime 2}}\right) d \varphi .
$$

The self-similar substitution

$$
R(\varphi, t)=\left(t^{*}-t\right)^{\beta} r(\varphi), \quad Y(\varphi, t)=\left(t^{*}-t\right)^{\beta} y(\varphi)
$$

does not change the meaning of the angle coordinate $\varphi$. It leads us to the following pair of equations for the functions $r(\varphi)$ and $y(\varphi)$ :

$$
\begin{aligned}
r^{2} & =\frac{\sqrt{r^{2}+r^{\prime 2}}}{y^{1-\alpha}} \\
y r & =\frac{1}{\alpha}\left(\frac{y^{\alpha} r^{\prime}}{\sqrt{r^{2}+r^{\prime 2}}}\right)^{\prime}-\frac{1}{\alpha} \frac{r y^{\alpha}}{\sqrt{r^{2}+r^{\prime 2}}} .
\end{aligned}
$$

We observe that there is no explicit dependence on $\varphi$ in these equations. This property helps us to integrate the system. The general solution can be represented in the following parametric form (see Appendix B for a detailed derivation):

$$
\begin{aligned}
& \varphi(p)=\varphi_{0}+\arctan (p)-\sqrt{\frac{\alpha(1-\alpha)}{(2-\alpha)(1+\alpha)}} \cdot \arctan \left(p \sqrt{\frac{\alpha(2-\alpha)}{\left(1-\alpha^{2}\right)}}\right) \\
& y(p)=C^{-\frac{1}{2-\alpha}}\left(\frac{\left(1-\alpha^{2}\right)}{\alpha(2-\alpha)}+p^{2}\right)^{\frac{1}{2(2-\alpha)}} \\
& r(p)=C^{\frac{1-\alpha}{2-\alpha}}\left(\frac{\left(1-\alpha^{2}\right)}{\alpha(2-\alpha)}+p^{2}\right)^{\frac{\alpha-1}{2(2-\alpha)}} \sqrt{1+p^{2}},
\end{aligned}
$$

where the parameter $p$ runs between the limits $-\infty<p<+\infty, C$ and $\varphi_{0}$ are arbitrary integration constants. The constant $C$ determines the asymptotic slope of the curve at large distances from the origin: $y \approx r / C$ when $r \rightarrow \infty$, while the constant $\varphi_{0}$ reflects the mentioned symmetry of the system with respect to rotations in $x$-z plane. The condition (35) for applicability of the local approximation (34) is satisfied if $C \sqrt{\alpha} \gg 1$. A typical self-similar solution $x(z)$ is shown in Fig.3. 


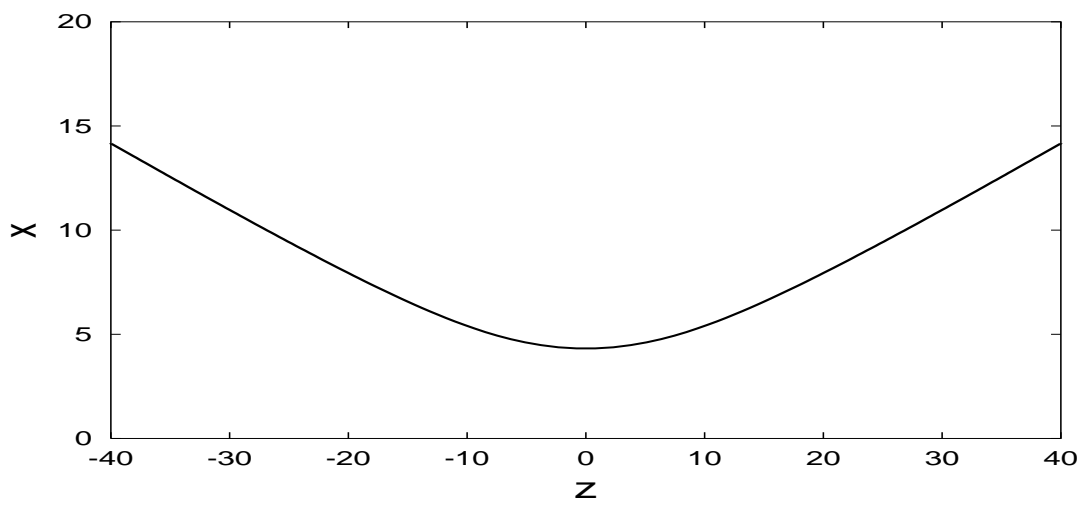

Figure 3: Self-similar solution $x(z)$ for $C=50, \alpha=0.1$.

It is interesting to note that the total angle $\Delta \varphi$ between two asymptotic directions in $x-z$ plane does not depend on the parameter $C$ in the long-scale local approximation used above:

$$
\Delta \varphi=\pi\left(1-\sqrt{\frac{\alpha(1-\alpha)}{(2-\alpha)(1+\alpha)}}\right) .
$$

At small values of $\alpha$, this angle approaches $\pi$. Another remark about $\Delta \varphi$ is that the above expression assumes identical values at $\alpha$ and at $1-\alpha$, so the value $\tilde{\alpha}=1 / 2$ results in the extremum $\Delta \varphi_{\min }=2 \pi / 3$. For this case, the curve lies on the cone $y=r / C$.

\section{Discussion}

We observed that in the systems (34) with $0<\alpha<1$, finite time singularity formation is possible in the self-similar regime. Inasmuch as the condition (35) for applicability of the approximate Hamiltonian (34) is satisfied in a range of the parameter $C$ related to the self-similar solutions (50-52), we conclude that in the systems (2) self-similar collapse of two symmetrical singular vortex filaments can also take place. The principal question is whether this is also possible for filaments having finite width. If yes, then such solutions are analogous to the assumed self-similar solutions of the Euler equation. Though the exponent $\beta$ (40) differs from $1 / 2$, the difference is small if $\alpha$ is small. However, an important difference exists between infinitely thin filaments and filaments with finite width: inside the latter, longitudinal flows take place, caused by a twist of the vortex lines constituting the filament. Those flows keep the width homogeneous along the filament if a local stretching is not sufficiently fast. This mechanism acts against singularity formation and, probably, in some cases it can prevent a singularity at all. [It is worth mentioning here that for finite width vortex structures in the Navier-Stokes equation frame, the usual "outcome" result of the Crow instability is vortex line reconnection [34.] Thus, a more-less consistent analysis of the general situation should take into account, besides the dynamics of a mean shape of the filament, at least the dynamics of the width and the conjugated dynamics of the twist. Clearly, we do not need to consider $\alpha \neq 0$ systems, when we deal with non-singular vortex filaments. It should be emphasized that an attempt to take account of finite width of the 
filament by simply using regularized Green's functions such as $G_{\epsilon}(r) \sim 1 / \sqrt{r^{2}+\epsilon^{2}}$ with a constant $\epsilon$, giving correct results for long scale limit of the linearized problem, fails to describe the dynamics in the highly nonlinear regime.

Also, we would like to note that a local approximation analogous to (34) is possible for arbitrary Green's function $G_{M}(r)$. The corresponding long scale Hamiltonian has the form

$$
\mathcal{H}_{M l}\{\mathbf{R}(\xi)\}=\oint F_{M}(Y) \sqrt{X^{\prime 2}+Z^{\prime 2}} d \xi,
$$

where the (positive) function $F_{M}(Y)$ is related to the function $G_{M}(r)$ in the following way:

$$
F_{M}(Y)=\int_{0}^{+\infty}\left(G_{M}(\xi)-G_{M}\left(\sqrt{\xi^{2}+(2 Y)^{2}}\right)\right) d \xi .
$$

The stationary motion with a constant coordinate $Y_{0}=b / 2$ is unstable if the second derivative of the function $F_{M}$ is negative at that value: $F_{M}^{\prime \prime}(b / 2)<0$. We believe that such systems can exhibit locally self-similar collapse, if the asymptotics of the function $F_{M}(Y)$ is power-like at small $Y: F_{M} \sim Y^{\alpha}$, with $0<\alpha<1$.

The final remark concerns the possibility of including effects caused by the fact that the unstable range is finite in the wave-number space into the approximate long scale theory. Especially this is important for the case of small values of $\alpha$, because in that limit the condition (35) for applicability of the Hamiltonian (34) becomes too restrictive. The idea how to improve the approximation is the following. In general, the exact expression for the Hamiltonian of a pair of singular filaments, after integration by parts, can be represented as the half of the integral over a surface $\Sigma$ drawn between the filaments (one half since we consider only one from two symmetric strings):

$$
\mathcal{H}_{\alpha}=\frac{1}{2} \int \frac{(\mathbf{v} \cdot \mathbf{p})}{2} d \mathbf{r}=\frac{\Gamma}{2} \int_{\Sigma} \frac{(\mathbf{v} \cdot d \mathbf{S})}{2},
$$

because the canonical momentum field $\mathbf{p}$ created by filaments is determined by a multivalued scalar potential $\Phi(\mathbf{r}): \mathbf{p}=\nabla \Phi$, which has the additive increment $\Gamma=\oint(\mathbf{p} \cdot d \mathbf{l})$ after passing around a filament. Also the equality $\operatorname{div} \mathbf{v}=0$ is important for derivation of the last expression. In the case of small $\alpha$, we should just more carefully take account of the contribution to the surface integral from the vicinity of filaments. As the result of such consideration, we find that for a better approximation it is sufficient to replace in (34) the projection of the arc-length element by the entire arc-length element and, correspondingly, use the Hamiltonian

$$
\mathcal{H}_{l}^{\alpha \ll 1}\{\mathbf{R}(\xi)\} \sim \oint \frac{Y^{\alpha}}{\alpha} \sqrt{X^{\prime 2}+Y^{\prime 2}+Z^{\prime 2}} d \xi .
$$

We stress once more here that this expression is valid only in the case $\alpha \ll 1, Y / L \ll 1$.

\section{Acknowledgments}

These investigations were supported by the Graduate School in Nonlinear Science (The Danish Research Academy), and by the INTAS. The work of V.P.R. was supported by the RFBR (grant No. 00-01-00929) and by the Russian State Program of Support of the Leading Scientific Schools (grant No. 00-15-96007). 


\section{Appendix A}

In order to have some closed expressions for the functions $A_{\alpha}(k)$ and $B_{\alpha}(k)$ instead of the integral representations (24) and (25), let us use the following mathematical relations [35]:

$$
\begin{gathered}
I_{n-\alpha}=\int_{0}^{+\infty} \frac{d \zeta}{\left(\zeta^{2}+1\right)^{\frac{n-\alpha}{2}}}=\frac{\sqrt{\pi}}{2} \frac{\Gamma\left(\frac{n-1-\alpha}{2}\right)}{\Gamma\left(\frac{n-\alpha}{2}\right)}, \\
I^{(1)}=\int_{0}^{+\infty} \cos (k b \zeta) \frac{d \zeta}{\zeta^{1-\alpha}}=(b k)^{-\alpha} \cos \left(\frac{\pi \alpha}{2}\right) \Gamma(\alpha), \\
I^{(3)}=\int_{0}^{+\infty}(1-\cos (k b \zeta)) \frac{d \zeta}{\zeta^{3-\alpha}}=\frac{(k b)^{2} I^{(1)}}{(1-\alpha)(2-\alpha)}, \\
\int_{0}^{+\infty} \frac{\cos (q \zeta) d \zeta}{\left(\zeta^{2}+1\right)^{\rho}}=\frac{\sqrt{\pi}}{\Gamma(\rho)}\left(\frac{q}{2}\right)^{\rho-\frac{1}{2}} K_{\rho-\frac{1}{2}}(q), \quad \rho>0,
\end{gathered}
$$

where $\Gamma(x)$ is the Gamma function, $K_{\nu}(x)$ is the modified Bessel function of the second kind. The integral (58) results in the equalities

$$
\begin{aligned}
& J^{(1)}=\int_{0}^{+\infty} \frac{\cos (k b \zeta) d \zeta}{\left(\zeta^{2}+1\right)^{\frac{1-\alpha}{2}}}=\frac{\sqrt{\pi}}{\Gamma\left(\frac{1-\alpha}{2}\right)}\left(\frac{k b}{2}\right)^{-\frac{\alpha}{2}} K_{-\frac{\alpha}{2}}(k b), \\
& J^{(3)}=\int_{0}^{+\infty} \frac{\cos (k b \zeta) d \zeta}{\left(\zeta^{2}+1\right)^{\frac{3-\alpha}{2}}}=\frac{\sqrt{\pi}}{\Gamma\left(\frac{3-\alpha}{2}\right)}\left(\frac{k b}{2}\right)^{1-\frac{\alpha}{2}} K_{1-\frac{\alpha}{2}}(k b), \\
& J^{(5)}=\int_{0}^{+\infty} \frac{\cos (k b \zeta) d \zeta}{\left(\zeta^{2}+1\right)^{\frac{5-\alpha}{2}}}=\frac{\sqrt{\pi}}{\Gamma\left(\frac{5-\alpha}{2}\right)}\left(\frac{k b}{2}\right)^{2-\frac{\alpha}{2}} K_{2-\frac{\alpha}{2}}(k b) .
\end{aligned}
$$

Thus, we have from (24) and (25):

$$
\begin{gathered}
A_{\alpha}(k)=2(1-\alpha)^{2} b^{\alpha-2} I^{(3)}-2 k^{2} b^{\alpha} J^{(1)}+2(1-\alpha) b^{\alpha-2}\left(I_{3-\alpha}-J^{(3)}\right), \\
B_{\alpha}(k)=2(1-\alpha)^{2} b^{\alpha-2} I^{(3)}-2(1-\alpha)(3-\alpha) b^{\alpha-2}\left(2\left(J^{(5)}+I_{5-\alpha}\right)-J^{(3)}-I_{3-\alpha}\right) .
\end{gathered}
$$

\section{Appendix B}

In this Appendix, we explain how the solution (50-52) of the system (4849) can be obtained. Let us introduce the designations

$$
Q=(d r / d \varphi)^{2}, \quad s=r^{2}
$$

then consider temporary $s$ as independent variable, and rewrite Eq.(49) as follows:

$$
y=\frac{2}{\alpha}\left(Q \frac{d}{d s}\left(\frac{y^{\alpha}}{\sqrt{s+Q}}\right)+\frac{y^{\alpha}(d Q / d s-1)}{2 \sqrt{s+Q}}\right),
$$


or equivalently

$$
y=\frac{2}{\alpha}\left(\alpha \frac{d y}{d s} y^{\alpha-1} \sqrt{s+Q}-\frac{d}{d s}\left(\frac{y^{\alpha} s}{\sqrt{s+Q}}\right)\right) .
$$

Substituting into this equation the relation

$$
Q=s\left(s y^{2(1-\alpha)}-1\right)
$$

which follows from the equation (48), we have the following equation for $y(s)$ :

$$
y=\frac{2}{\alpha} \frac{d y}{d s}\left(\alpha s+(1-2 \alpha) y^{2(\alpha-1)}\right) .
$$

This first order differential equation is linear for the inverse dependence $s(y)$, and its general solution is

$$
s(y)=C^{2} y^{2}-\frac{(1-2 \alpha)}{\alpha(2-\alpha)} y^{2(\alpha-1)},
$$

where $C$ is an arbitrary constant of integration. Thus, we have the relation between $y$ and $s=r^{2}$. To obtain another relation, between $y$ and $\varphi$, let us use the equation

$$
d \varphi=\frac{d s}{2 \sqrt{s Q}},
$$

which gives us the integral

$$
\begin{gathered}
\varphi-\varphi_{0}=\int \frac{s^{\prime}(y) d y}{2 s(y) \sqrt{s(y) y^{2(1-\alpha)}-1}}=\int \frac{\left(C^{2} y^{2(2-\alpha)}+\frac{(1-2 \alpha)(1-\alpha)}{\alpha(2-\alpha)}\right) \frac{d y}{y}}{\left(C^{2} y^{2(2-\alpha)}-\frac{(1-2 \alpha)}{\alpha(2-\alpha)}\right) \sqrt{C^{2} y^{2(2-\alpha)}-\frac{\left(1-\alpha^{2}\right)}{\alpha(2-\alpha)}}}= \\
=\arctan \sqrt{\left(C^{2} y^{2(2-\alpha)}-\frac{\left(1-\alpha^{2}\right)}{\alpha(2-\alpha)}\right)}-\sqrt{\frac{\alpha(1-\alpha)}{(2-\alpha)(1+\alpha)} \arctan \sqrt{\left(\frac{\alpha(2-\alpha)}{\left(1-\alpha^{2}\right)} C^{2} y^{2(2-\alpha)}-1\right)} .}
\end{gathered}
$$

After introducing the new parameter

$$
p=\sqrt{\left(C^{2} y^{2(2-\alpha)}-\frac{\left(1-\alpha^{2}\right)}{\alpha(2-\alpha)}\right)},
$$

we arrive at solution of the system (48-49) in the form (50 52).

\section{References}

[1] J.T. Beale, T. Kato and A. Majda, Commun. Math. Phys. 94, 61 (1984).

[2] P. Constantin and C. Fefferman, Indiana Univ. Math. J. 42, 775 (1993).

[3] P. Constantin, C. Fefferman and A. Majda, Commun. Partial Diff. Equat. 21 559, (1996). 
[4] A. Pumir and E. D. Siggia, in Topological Fluid Mechanics, edited by H. K. Moffatt and A. Tsinober (Cambridge Univ. Press, Cambridge, 1990), p.469 ; Phys. Fluids A 4, 1472 (1992).

[5] M.E. Brachet et al., Phys. Fluids A 4, 2845 (1992).

[6] R.M. Kerr, Phys. Fluids 5, 1725 (1993).

[7] O.N. Boratav and R.B. Pelz, Phys. Fluids 6, 2757 (1994).

[8] U. Frish, Turbulence. The Legacy of A.N. Kolmogorov (Cambridge Univ. Press, Cambridge, 1995).

[9] R.B. Pelz, Phys. Rev. E 55, 1617 (1997).

[10] R. Grauer, C. Marliani and K. Germaschewski, Phys. Rev. Lett. 80, 4177 (1998).

[11] V.E. Zakharov, in Nonlinear MHD Waves and Turbulence, edited by T.Passot and P.L.Sulem, Lecture Notes in Physics, Vol. 536, (Springer, Berlin, 1999), pp. 369-385.

[12] K. Ohkitani and J.D. Gibbon, Phys. Fluids 12, 3181 (2000).

[13] H.K. Moffatt, J. Fluid Mech. 409, 51 (2000).

[14] V. Berdichevsky, Phys. Rev. E 57, 2885 (1998).

[15] E.A. Kuznetsov and V.P. Ruban, JETP Letters 67, 1076 (1998); Phys. Rev. E 61, 831 (2000).

[16] V.P. Ruban, JETP 89, 299 (1999).

[17] V.P. Ruban, Phys. Rev. E 62, 4950 (2000).

[18] S.C. Crow, AIAA J. 8, 2172 (1970).

[19] V.I. Arnol'd, Mathematical Methods of Classical Mechanics, 2nd edition (SpringerVerlag, New York, 1989).

[20] B.A. Dubrovin, S.P. Novikov, and A.T. Fomenko, Modern Geometry (Nauka, Moscow, 1979).

[21] R. Salmon, Ann. Rev. Fluid Mech. 20, 225 (1988).

[22] N. Padhye and P.J. Morrison, Plasma Phys. Rep. 22, 869 (1996).

[23] V.E. Zakharov and E.A. Kuznetsov, Phys. Usp. 40, 1087 (1997).

[24] P. J. Morrison, Rev. Mod. Phys. 70, 467 (1998).

[25] V.I. Il'gisonis and V.P. Lakhin, Plasma Phys. Rep. 25, 58 (1999). 
[26] V.P. Ruban, arXiv:physics/0007010.

[27] D.D. Holm, J.E. Marsden, and T.S. Ratiu, arXiv:chao-dyn/9801015.

[28] D.D. Holm et al., arXiv:chao-dyn/9903036.

[29] A.Chorin, J. Fluid Mech. 57, 785 (1973).

[30] M. Oliver and S. Shkoller, arXiv:math.AP/9910088.

[31] B.T. Nadiga and S. Shkoller, arXiv:math.NA/0005035.

[32] M.I. Monastyrskii and P.V. Sasorov, Sov. Phys. JETP 66, 683 (1987).

[33] A. Ruzmaikin and P. Akhmetiev, Phys. Plasmas 1, 331 (1994).

[34] S. Kida and M. Takaoka, Annu. Rev. Fluid Mech., 26, 169 (1994).

[35] A. P. Prudnikov, Yu. A. Brychkov, O. I. Marichev, Integrals and Series. Elementary Functions (Nauka, Moscow, 1981) [in Russian]; I. S. Gradshteyn and I. M. Ryzhik, Table of Integrals, Series, and Products, 4th edition (Academic Press, Inc., Orlando, San Diego, New York, London, Toronto, Monreal, Sydney, Tokyo, 1980). 